\title{
BAND WEIGHTING AND SELECTION BASED ON HYPERPLANE MARGIN MAXIMIZATION FOR HYPERSPECTRAL IMAGE CLASSIFICATION
}

\author{
Cheng Yan ${ }^{1}$, Xiao Bai ${ }^{1}$, Jun Zhou $^{2}$ \\ ${ }^{1}$ School of Computer Science and Engineering, Beihang University \\ Haidian District, Beijing, China \\ ${ }^{2}$ School of Information and Communication Technology, Griffith University \\ Nathan, QLD 4111, Australia
}

\begin{abstract}
Band selection is an effective solutions for dimensionality reduction in hyperspectral imagery. In this paper, a novel band weighting and selection method is proposed based on maximizing margin in support vector machine (SVM). The goal is to reduce high dimensionality if hyperspectral data while achieving accuracy classification performance. This method computes the weights of the samples to maximize the margin between the samples and the hyperplane in SVM. Bands are selected if they can enlarge the differences between classes and improve the classification performance. Experiments on two public benchmark hyperspectral datasets show the effectiveness of our method.
\end{abstract}

Index Terms- Band weighting and selection, support vector machine, hyperspectral Imagery, classification

\section{INTRODUCTION}

Hyperspectral imagery has been used in a variety of remote sensing applications. However, its large data volume leads to challenging problems, such as high computation complexity and Hughes phenomenon [1]. Dimensionality reduction is a solution to mitigate these problems. Many methods in this area transforms hyperspectral data from their original space to a target lower dimensional space. Along this line, typical methods include projection pursuit(PP) [2], principal component analysis (PCA) [3], and independent component analysis (ICA) [4]. However, such transformation based methods change the physical meaning of the original spectral data.

Band selection is another kind of solution which removes a subset of original bands, hence maintaining the physical meaning of remaining bands. The band selection method$\mathrm{s}$ can be further divided into two types, supervised and unsupervised [5]. Unsupervised methods are employed to select the most distinctive and informative bands. Pabitra et al. [6] described an unsupervised feature selection algorithm based on measuring the similarity between features. Cariou et al. [7] proposed a unsupervised method using an information theoretic criterion to automatically separate sensor's spectral range into disjoint sub-bands. Being convenient to implemen$\mathrm{t}$, unsupervised methods still can not match the supervised methods in image classification accuracy [8].

Supervised band methods use training data with labels to establish a predictive model for band selection. In [9], Song et al. proposed a backward elimination procedure for feature selection using the Hilbert-Schmidt Independence Criterion. Yang et al. [10] proposed a supervised band selection algorithm that uses the known class signatures only without examining the original bands. More recently, Ly et al. [11] used a sparse graph to construct a supervised dimensionality reduction method. This is referred to as sparse graph-based discriminant analysis. However, these methods do not give full consideration on the discrimination between the classes, which limits its classification accuracy.

In this paper, we present a supervised band weighting and selection method which is based on maximizing the margin$\mathrm{s}$ between samples and the hyperplane in the support vector machine (SVM) framework. We assign a vector to each class to weight the training samples. After maximizing the classification score on the training set, the optimal weight vector is obtained and the bands with the highest weights are selected. These selected bands keep the information revealing the difference between classes which is useful for classification. The contributions of this paper are two-fold. Firstly, we maximize the classification score of each sample to maximize the margin in the SVM framwork. By weighting these samples and selecting the bands with highest weights, our method not only keeps the physical meaning of the original bands but also takes full advantage of the training set to discriminate the class difference. Secondly, the weight vector reveals the importance of bands in which differences between classes are enlarged so that the bands with the highest values reflect the most important information in classification. We used two public benchmark hyperspectral datasets to validate the effectiveness of our method.

The rest of the paper is organized as follows. The proposed method is described in Section 2. Section 3 and Section 4 are dedicated to experiment and conclusions, respec- 
tively.

\section{APPROACH}

In the proposed method, let $x_{i}$ be an $N$-dimensional sample with corresponding label $y_{i}$. In a general classification problem with SVM [12] as the classifier, the objective is to minimize the following equation

$$
\min \frac{1}{2}\|w\|^{2}+C \sum \max \left(0,1-y_{i} f\left(w, x_{i}\right)\right)
$$

The goal of training is to estimate parameter $w$ of the classifier. Considering a multi-class one-vs-all setting, when all classifiers are obtained, each for a training class, we can predict which class a test sample belongs to by looking into the value of $w_{i}^{T} x$ (details in [12]). In the proposed approach, a weight vector $v(n \times 1)$ is created for each class to reveal the importance of bands, then bands with the highest weights are selected for classification. The process is as follows. For each class, we first train an initial classifier by using samples from this class as positive data, and samples from other classes as negative data. Then we weight training samples to maximize their classification scores which is equivalent to enlarge the margin of SVM. After optimization, $v$ is obtained that reveals the importance of bands for each class. Finally, we select the bands with the highest weights and use corresponding information of training set to re-train the classifier. These selected bands keep the information revealing the difference between classes which is useful for classification. We adopt the same procedure to train each class. Note that for each class, selected bands may be different. When a test sample comes in, we feed it into every classifier with corresponding selected bands. It belongs to a the class with the highest score.

\subsection{Initial classifier}

We first train an initial classifier in which the hyperplane $w$ will be obtained. The optimization target is Eq. (1). It is the same as the optimization problem of SVM. $C$ is a parameter to punish the error. We use the standard optimization approach to calculate $w$ [12].

\subsection{Band Selection}

We expect to make the score of positive samples higher and the score of negative samples lower, which is equivalent to expand the margin between samples and the corresponding hyperplane. To achieve this goal, we first assign a weight vector to the samples. To avoid trivial solution, we add constraint to control the deviation from $v$ and a uniform vector. Therefore, the objective becomes

$$
\max \sum y_{i} w^{T}\left(v \times x_{i}\right)-\beta\|v-1\|^{2}
$$

where $\left(v \times x_{i}\right)$ is the dot product between $v$ and $x_{i}$, and $y_{i}$ is the label of $x_{i}$. The second term is contraint term and the parameter $\beta$ controls the deviation of the weight vector $v$ from a uniform vector. When $\beta$ is large, this term forces all weighting vector to be the same at each band, thus, the original spectral data is mostly maintained with slight tuning by $v$ for classification. On the contrary, when $\beta$ is small, the resulting weights may differ significantly for each band. The weight vector $v$ reflects the importance of each band. A larger value means the corresponding band is important as it is highly discriminating for a particular class. We select the bands with the highest weight value. Finally, we retrain the classifier through Eq. (1) using the training set with the selected bands.

The pseudo-code of the algorithm is presented in Table 1.

Table 1. The Pseudo-CODE OF THE PROPOSED AlgoRITHM

Algorithm

Input: training set $X=x_{i}$ with label $Y=y_{i}$

Output: selected bands and the classifier parameter $w$

for Each class $i=1 \cdots N$

1. Train the initial classifier

2. Optimize $v$ through Eq. (2)

3. Re-train the classifier with selected bands

end for

\section{EXPERIMENTAL RESULTS}

In this section, we describe the experimental results on two benchmark datasets. The first dataset was acquired by the AVIRIS sensor over Indian Pines of northwest Indiana in 1992. It has been reduced from the original 220 bands to 200 bands by discarding the noisy bands. In the experiment, 10 percent samples of each class were randomly selected as the training set. The parameter $\beta$ was set to 10 percent of the number of samples selected for training the corresponding classifier. Table 1 shows the number of training and testing samples.

We compare our method with three other band selection methods, i.e., MVPCA [13], AP [14], and MEAC [10]. MVP$\mathrm{CA}$ ranks bands based on the importance of individual band and its correlation with other bands. AP is a band selection method using affinity propagation. MEAC uses the known class signatures only without examining the original bands or the need of class training samples. The results from our method are displayed in Fig. 1. To investigate the impact of band selection on the classification accuracy, we changed the number of bands from 5 to 40 by selecting bands with the 
highest weights. The results of all methods under comparison are shown in Fig. 2.

Table 2. Number of tRAining AND testing SAMPLES FOR EACH OBJECT CLASS.

\begin{tabular}{|c|c|c|}
\hline CLASS & TRAINING & TES \\
\hline C1 - Alfalfa & 5 & \\
\hline C2 - Corn-notill & 142 & \\
\hline C3 - Corn-mintill & 83 & \\
\hline C4 - Corn & 23 & \\
\hline C5 - Grass-pasture & 48 & \\
\hline C6 - Grass-trees & 73 & \\
\hline C7 - Grass-pasture-mowed & 3 & \\
\hline C8 - Hay-windrowed & 47 & \\
\hline C9 - Oats & 2 & \\
\hline C10 - Soybean-notill & 97 & \\
\hline C11 - Soybean-mintill & 245 & \\
\hline C12 - Soybean-clean & 59 & \\
\hline C13 - Wheat & 20 & \\
\hline C14 - Woods & 126 & \\
\hline C15 - Buildings-Grass-Trees-Drives & 38 & \\
\hline C16 - Stone-Steel-Towers & 9 & \\
\hline TOTAL & 1020 & \\
\hline
\end{tabular}

Fig. 1. Indian Pines data and classification results. The lefthand side is a sample band of Indian Pines dataset. The righthand side is the classification results of our method.

The second dataset is Salinas scene which was collected by the AVIRIS sensor over Salinas Valley, California. It contains 224 bands over 0.4 to $2.5 \mathrm{~m}$, with 16 classes and $3.7 \mathrm{~m}$ spatial resolution. It has been reduced from the original 220 bands to 200 bands by removing the noisy bands. We also randomly selected 5 percent samples from each class to form the training set and set $\beta$ to 10 percent of number of training samples. Table 3 shows the number of training and testing samples in this data set. Fig. 3 shows the results of our method, with comparison against alternative methods being displayed in Fig. 4.

It is clear that our method has outperformed the alternatives, especially when more than 15 bands are selected. The advantage of our method is because we take each training sample into consideration to find the bands with highly discriminative information. It is effective to improve classifica-

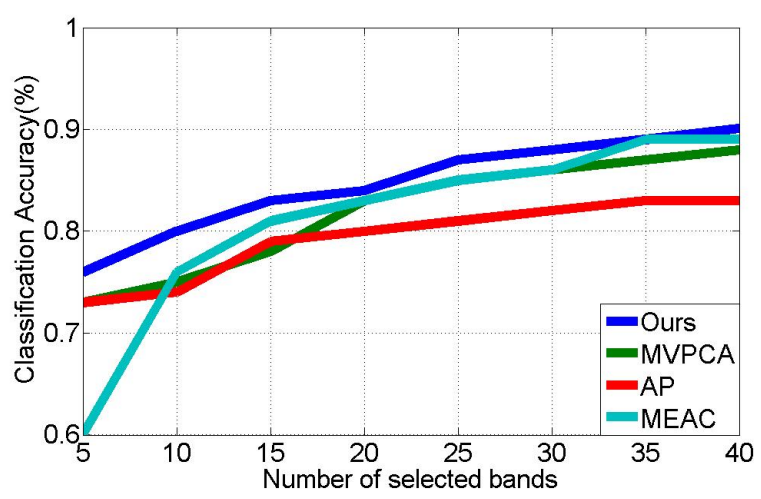

Fig. 2. Comparison of classification accuracy on the Indian Pines data, when different number of bands are selected.

Table 3. Number of tRAining AND testing SAMPles FOR EACH OBJECT CLASS

\begin{tabular}{l|c|c}
\hline \hline CLASS & TRAINING & TESTING \\
\hline \hline C1 - Broccoli-1 & 100 & 2000 \\
C2 - Broccoli-2 & 175 & 3500 \\
C3 - Fallow & 85 & 1700 \\
C4 - Fallow-rough-plow & 55 & 1100 \\
C5 - Fallow-smooth & 125 & 2500 \\
C6 - Stubble & 195 & 3900 \\
C7 - Celery & 175 & 3500 \\
C8 - Grapes-untrained & 500 & 10000 \\
C9 - Sell-vineyard-develop & 275 & 5500 \\
C10 - Corn-senesced-green-weeds & 150 & 3000 \\
C11 - Lettuce-romain-4-weeks & 45 & 900 \\
C12 - Lettuce-romain-5-weeks & 90 & 1800 \\
C13 - Lettuce-romain-6-weeks & 40 & 800 \\
C14 - Lettuce-romain-7-weeks & 40 & 800 \\
C15 - Vineyard-untrained & 325 & 6500 \\
C16 - Vineyard-vertical-trellis & 75 & 1500 \\
\hline TOTAL & 2450 & 49000 \\
\hline
\end{tabular}

tion result.

\section{CONCLUSION}

In this paper, we have introduced a new hyperspectral band weighting and selection method which is based on maximizing the margin in support vector machine. In this method, we first train initial classifiers. Then we weight samples and maximize the value of score of each sample to make the margin larger in SVM framework. The weight vector reflects the importance of each band, which allows us choose the bands with the highest value for classification. Experimental evaluation shows that the proposed method has better performance than several state-of-the-arts methods. 

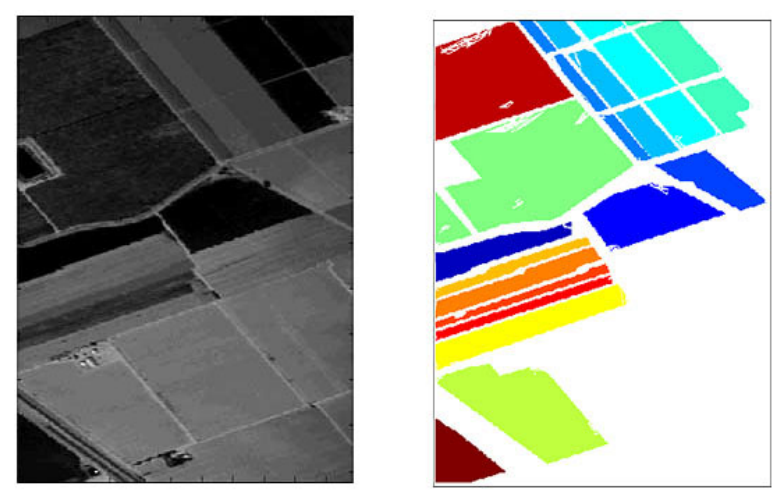

Fig. 3. Salinas Data and classification results from the proposed method. The left-hand side is a sample band of Salinas dataset. The right-hand side shows the classification result of the proposed method.

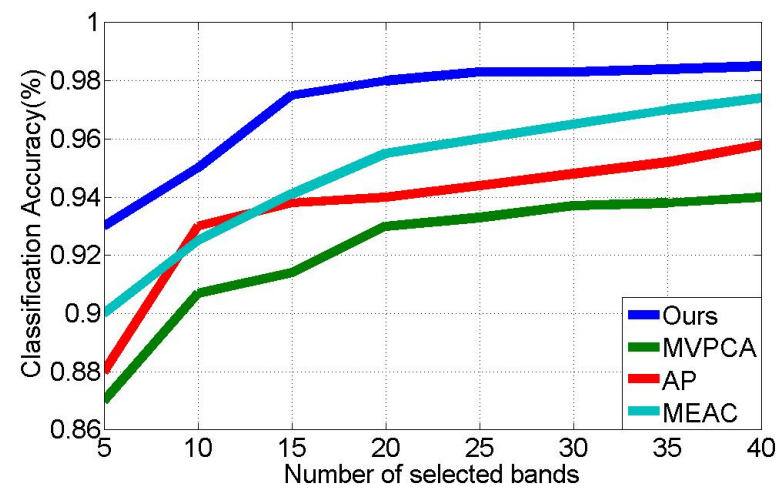

Fig. 4. Comparison of classification accuracy on the Salinas data, when different number of bands are selected..

\section{ACKNOWLEDGE}

This work is supported by China NSFC Project No. 61105022 and Australian Research Council's DECRA Projects funding scheme ID DE120102948.

\section{REFERENCES}

[1] Gordon Hughes, "On the mean accuracy of statistical pattern recognizers," Information Theory, IEEE Transactions on, vol. 14, no. 1, pp. 55-63, 1968.

[2] Agustin Ifarraguerri and Chein-I Chang, "Unsupervised hyperspectral image analysis with projection pursuit," Geoscience and Remote Sensing, IEEE Transactions on, vol. 38, no. 6, pp. 2529-2538, 2000.

[3] Abhishek Agarwal, Tarek El-Ghazawi, Hesham ElAskary, and Jacquline Le-Moigne, "Efficient hierarchical-pca dimension reduction for hyperspectral imagery," in Signal Processing and Information Technology, 2007 IEEE International Symposium on. IEEE, 2007, pp. 353-356.

[4] Qian Du and He Yang, "Similarity-based unsupervised band selection for hyperspectral image analysis," Geoscience and Remote Sensing Letters, IEEE, vol. 5, no. 4, pp. 564-568, 2008.

[5] Peter Bajcsy and Peter Groves, "Methodology for hyperspectral band selection," Photogrammetric Engineering \& Remote Sensing, vol. 70, no. 7, pp. 793-802, 2004.

[6] Pabitra Mitra, CA Murthy, and Sankar K. Pal, "Unsupervised feature selection using feature similarity," IEEE transactions on pattern analysis and machine intelligence, vol. 24, no. 3, pp. 301-312, 2002.

[7] Claude Cariou, Kacem Chehdi, and Steven Le Moan, "Bandclust: an unsupervised band reduction method for hyperspectral remote sensing," Geoscience and Remote Sensing Letters, IEEE, vol. 8, no. 3, pp. 565-569, 2011.

[8] Manoranjan Dash, Hua Liu, and Jun Yao, "Dimensionality reduction of unsupervised data," in Tools with Artificial Intelligence, 1997. Proceedings., Ninth IEEE International Conference on. IEEE, 1997, pp. 532-539.

[9] Le Song, Alex Smola, Arthur Gretton, Karsten M Borgwardt, and Justin Bedo, "Supervised feature selection via dependence estimation," in Proceedings of the 24th international conference on Machine learning. ACM, 2007, pp. 823-830.

[10] He Yang, Qian Du, Hongjun Su, and Yehua Sheng, "An efficient method for supervised hyperspectral band selection," Geoscience and Remote Sensing Letters, IEEE, vol. 8, no. 1, pp. 138-142, 2011.

[11] Nam Hoai Ly, Qian Du, and James E Fowler, "Sparse graph-based discriminant analysis for hyperspectral imagery," 2014.

[12] Chih-Chung Chang and Chih-Jen Lin, "Libsvm: a library for support vector machines," ACM Transactions on Intelligent Systems and Technology (TIST), vol. 2, no. 3 , pp. 27, 2011.

[13] Chein-I Chang, Qian Du, Tzu-Lung Sun, and Mark L$\mathrm{G}$ Althouse, "A joint band prioritization and banddecorrelation approach to band selection for hyperspectral image classification," Geoscience and Remote Sensing, IEEE Transactions on, vol. 37, no. 6, pp. 26312641, 1999.

[14] Yuntao Qian, Futian Yao, and Sen Jia, "Band selection for hyperspectral imagery using affinity propagation," IET Computer Vision, vol. 3, no. 4, pp. 213-222, 2009. 